\title{
Crowded field 3D spectroscopy of LBV candidates in M 33
}

\author{
S. Fabrika ${ }^{1}$, O. Sholukhova ${ }^{1}$, T. Becker ${ }^{2}$, V. Afanasiev ${ }^{1}$, M. Roth ${ }^{2}$, and S. F. Sanchez ${ }^{2}$ \\ 1 Special Astrophysical Observatory, Nizhnij Arkhyz, Karachaevo-Cherkesia, 369167, Russia \\ e-mail: fabrika@sao.ru \\ 2 Astrophysikalisches Institut Potsdam, An der Sternwarte 16, 14482, Potsdam, Germany \\ e-mail: mmroth@aip.de
}

Received 9 December 2003 / Accepted 22 February 2005

\begin{abstract}
We present integral field spectroscopy of the LBV candidate stars B 416 and v 532 in the local group galaxy M 33 . B 416 is surrounded by an elongated ring-like nebula, which has a projected radius of $20 \times 30 \mathrm{pc}$. From the datacube we create ionization and radial velocity maps of the nebula. The excitation of the gas decreases towards the outer part of the ring, while the inner part of the nebula is filled with a more excited gas. In the EW direction the ring is seen to expand with a maximum projected velocity amplitude of about $40 \mathrm{~km} \mathrm{~s}^{-1}$. The eastern part approaches the observer. We estimate the nebula dynamical lifetime $\sim 8 \times 10^{5}$ years. It could be a residual MS bubble, which indicates a main-sequence or pre-LBV status of the star. We classify B 416 as an "iron star" or B[e]-supergiant. In v 532 an elongated nebula has been marginally detected. The total projected size of the nebula along the main axis is $30 \mathrm{pc}$, and the total radial velocity gradient is $44 \pm 11 \mathrm{~km} \mathrm{~s}^{-1}$. $\mathrm{v} 532$ exhibits both strong photometric and spectral variability. At the time of our observations it was in an intermediate brightness state with a rich nitrogen spectrum. We classify v 532 as an LBV, showing LBV $\leftrightarrow$ Ofpe/WN transitions. We stress the importance of integral field spectroscopy as the optimal technique for studying nebulae and the evolution of LBV-like stars in nearby galaxies.
\end{abstract}

Key words. Local Group - stars: early-type - stars: individual: v 532 - HII regions - stars: individual: B416 galaxies: individual: M33

\section{Introduction}

Analysis of the brightest supergiants as individual stars in external galaxies provides a unique tool for determining the properties of young stellar populations in their host galaxy. In particular, nearby galaxies are ideally suited to studying the most massive stars systematically, both during their normal evolution in different environments, as well as during the short and unstable stages of evolution as OB supergiants, rare hypergiants, Luminous Blue Variables (LBVs), Ofpe/WN stars, B[e]-supergiants (Kudritzki 1998; Lamers et al. 2001), or during their latest stages of evolution as peculiar massive binary objects with relativistic jets and accretion disks like SS 433 (Fabrika \& Sholukhova 1995). All of these stars are young and massive, and they are among the brightest stars in their host galaxy. They share some main spectral properties like a blue continuum and strong emission lines, and they often show a surrounding nebula. In the Milky Way a considerable fraction of such stars may be hidden by dust extinction in the galactic plane, which is why they are observed best in nearby galaxies.

The evolutionary connections between the various classes of massive stars, such as the OB-stars, B[e]-supergiants, LBVs, Ofpe/WNL, and Wolf-Rayet stars are not well understood. Systematic surveys of LBV-like candidates in massive galaxies (Calzetti et al. 1995; Massey et al. 1996; Corral 1996;
Fabrika \& Sholukhova 1999) provide a basis for isolating these objects for subsequent follow-up and quantative spectroscopy (Massey et al. 1996; Sholukhova et al. 1997, 1999) to reveal the basic properties of these objects. In M 33 there are 4 confirmed LBV stars and about a dozen LBV candidates, but only one known Ofpe/WN star, and about 5 new candidates for this latter class of objects (Massey et al. 1996). Not a single SS433-type star has been found in M33, and also no B[e]-supergiant has been confirmed to date.

LBVs are extremely massive stars $\left(M>40 M_{\odot}\right)$ at an unstable stage of evolution with a mass loss rate of $\sim 10^{-4} M_{\odot} / y$. They show strong spectral and photometrical variability on time-scales from months to years (Humphreys \& Davidson 1994; Lamers et al. 1998a). LBVs represent an instability stage after the main sequence. It is not clear whether there is a relation between LBVs and B[e]-supergiants (Conti 1976), since their spectra often look practically the same, but the latter stars do not show strong variability (Zickgraf et al. 1986; Lamers et al. 1998b). Spectral properties of B[e]-supergiants can be interpreted by a non-isotropical mass-loss occurring in the equatorial plane (Zickgraf et al. 1985; Zickgraf et al. 1986). They are probably a subclass of rapidly rotating post main-sequence stars. One may expect an evolutionary connection between the $\mathrm{B}[\mathrm{e}]$-supergiants and the LBVs. 
Important information can be derived from morphological studies of the nebulae surrounding such stars. Because of their heavy mass loss, LBV stars are expected to show extended envelopes, which consist of ejected matter and swept-up interstellar material. The same is true for $\mathrm{B}[\mathrm{e}]$ supergiant stars. Direct imaging and kinematic studies help to shed light on the mass loss history and on the physics of the stellar wind.

Numerical models of the nebular expansion around massive stars (Garcia-Segura et al. 1996) have shown that those nebulae reach diameters of up to $20-40 \mathrm{pc}$ at the main-sequence and pre-LBV stages. Such scales correspond to $\sim 10^{\prime \prime}$ at the distance of M 33 (3"'5/pc). LBV nebulae are smaller because of a shorter evolution time. Practically all known LBV stars have circumstellar shells (Humphreys \& Davidson 1994). Typical LBV nebula sizes are in the range of $0.1-4 \mathrm{pc}$, expansion velocities $15-100 \mathrm{~km} \mathrm{~s}^{-1}$, and dynamical times are in the range $100-5 \times 10^{4}$ years (Nota 1999; Figer et al. 1999; Weis 2003). There are possibly larger gas nebulae around LBV stars, for example a ring-like nebula around S Dor has a size up to $70 \mathrm{pc}$ (Weis 2003).

Spectroscopic analysis of the star is often hampered by the presence of strong nebular emission lines which contaminate the stellar spectrum, and is complicated in crowded fields. For our targets we choose the technique of Integral Field Spectroscopy (IFS, sometimes also called 3D Spectroscopy) in order to optimize the separation of the stars from their crowded environments. It provides both improved subtraction of nebular contamination from the stellar spectrum and spatially resolved nebular spectra. One can make extensive use of the full 2-dimensional information contained in 3D data, which can be also described as stacks of very narrow bandwidth filter images (datacubes).

Here we report on results of IFS observations of two LBV candidates in the Local Group galaxy M 33, the stars B 416 and $\mathrm{v} 532$. The first star was designated as blue star N416 in the photographic survey by Humphreys \& Sandage (1980). It was also identified as an LBV candidate in follow-up spectroscopy of the UV-brightest stars of M 33 (Massey et al. 1996). In a program searching for SS 433-like stars in M 33, Shemmer \& Leibowitz (1998) detected periodic microvariations in the photometry of B 416. These oscillations were confirmed as periodic and coherent by Shemmer et al. (2000), who classified the star as LBV based on the spectral similarity in comparison with typical LBV spectra at a quiescence stage. The coherent brightness oscillations may become quite important for our understanding of the physics of LBV stars; however, B 416 does not show the large amplitude variability typical for LBVs.

The second object of this paper is the variable blue star v 532 (Romano 1978; Artyukhina et al. 1995), which is known to exhibit the characteristic variability of LBVs, i.e. an amplitude of $\approx 1^{\mathrm{m}}$ on a time-scale of years (Kurtev et al. 2001). Shortscale and quasi-periodical variabilities have also been found in this star (Sholukhova et al. 2002). Strong emission lines, spectral and photometrical variability (Szeifert 1996; Fabrika 2000; Sholukhova et al. 2002; Polcaro et al. 2003) indicate that v 532 is an LBV object.

\section{Observations and data reduction}

Observations of v 532 and B 416 were carried out with the Multi-Pupil Fiber Spectrograph MPFS ${ }^{1}$ (Afanasiev 1998) at the prime focus of the $6 \mathrm{~m}$ telescope BTA (Russia). The integral field unit (IFU) of $16 \times 15$ square spatial elements ("spaxels") covers a rectangular region of $16^{\prime \prime} \times 15^{\prime \prime}$ on the sky ( $1^{\prime \prime}$ per element). Optical fibers transmit the light from the 240 spaxels of the IFU together with 16 additional fibers located at a radius of $\approx 4^{\prime} .5$ outside of the IFU for the purpose of sampling the sky background far away from the field. In nearby galaxies like in M33 the sky fibers will still be located within the galaxy, thus we did not use the sky fibers for the background subtraction. The entire set of 256 fibers is reformatted to form a pseudo-slit, whose emerging light is dispersed and projected onto the focal plane of the spectrograph. We used a SITe TK1024 backside-illuminated CCD with $1024 \times 1024$ pixels and a pixel size of $24 \mu \mathrm{m}$.

Two 20-min exposures of v 532 were taken on September 18,1998 with a reciprocal dispersion of $1.3 \AA$ Aixel $(F W H M \approx$ $3.5 \AA$ ) in the spectral range $4470-5800 \AA$ with a seeing of $1.5-2 \operatorname{arcsec} F W H M$. B 416 was observed on September 28, 1998 in two 20-min exposures with a reciprocal dispersion of $2.6 \AA /$ pixel $(F W H M \approx 7 \AA)$ in the spectral range of 4250 $6900 \AA$ with a seeing of $3 \operatorname{arcsec} F W H M$.

3D observations of B 416 were also carried out with INTEGRAL $^{2}$ at the $4.2 \mathrm{~m}$ WHT, La Palma (Arribas et al. 1998) on January 18, 2001. This instrument is a bare fiber bundle type of 3D spectrograph. Contrary to MPFS, the IFU has no lensarray in front of the fibers, resulting in a hexagonal package with small gaps between the circular fiber apertures. We used the SB1 bundle, which has 205 fibers and covers a rectangular FOV of $7.8 \times 6.4 \operatorname{arcsec}^{2}$ with a projected fiber diameter of $0{ }^{\prime} 45$. The fiber bundle is coupled to the bench-mounted fiber spectrograph WYFFOS on the Nasmyth platform of the WHT. The detector is a thinned, backside-illuminated $1 \mathrm{~K} \times 1 \mathrm{~K}, 24 \mu \mathrm{m}$ pixel CCD. We used an R300B grating, giving a spectral coverage of $5800 \AA$ and a spectral resolution of $11 \AA$ at a reciprocal dispersion of $5.8 \AA /$ pixel and took 2 exposures of $1800 \mathrm{~s}$ each. The conditions were clear with a seeing of 0.9-1.0" FWHM.

Data reduction for both the MPFS and INTEGRAL data sets was performed with the P3d package (Becker 2002), which was originally developed for the PMAS instrument (Roth et al. 2000), but has also been shown to work well for other instruments. The raw CCD frames were bias and dark subtracted, and cleaned from cosmic ray events. The spectra extraction was performed using a profile fitting algorithm, which simultaneously solves for cross-talk between adjacent spectra and straylight patterns on the detector. P3d incorporates a robust algorithm to reliably determine the geometry of the spectra. After extraction a wavelength calibration was applied using arc exposures. The wavelength-dependent fiber-to-fiber transmission variation was calibrated by means of twilight flatfield exposures. At this final stage of data reduction a 2-dimensional

\footnotetext{
1 http://www.sao.ru/ gafan/devices/mpfs/ mpfs_main.htm

2 http://www.ing.iac.es/ bgarcia/integral/html/ integral_home.html
} 

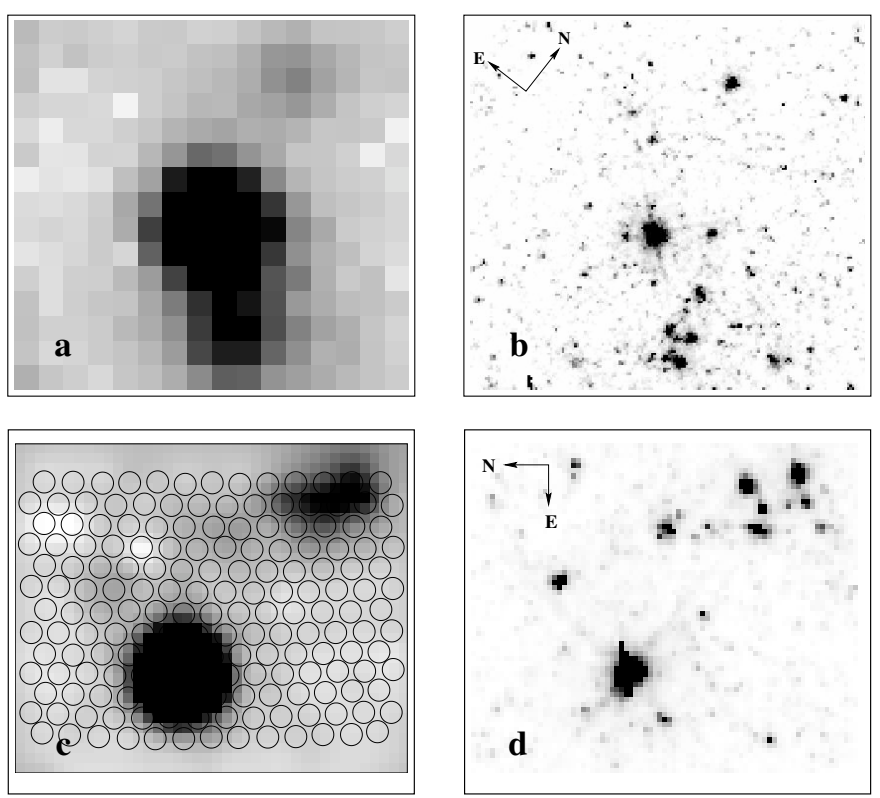

Fig. 1. a) MPFS map of B416 at $6000 \AA$ (spatial sampling 1 arcsec, the FOV is $16 \times 15 \operatorname{arcsec}^{2}$ ) and the same field b) from the HST WFC2 image. An enlarged fraction of this field from the INTEGRAL in the same wavelength c) and the corresponding enlarged HST field d). Note the different orientation of the MPFS and INTEGRAL fields.

image of stacked spectra appears. Monochromatic maps or an entire datacube may be subsequently derived from this stacked spectra format. The final analysis of the fully reduced 3D data, i.e. generation of monochromatic maps at selected wavelengths, co-adding flux within a digital aperture, sky subtraction, etc., was performed with the MONOLOOK tool (Becker 2002).

The full 2-dimensional spatial information allows one to accurately model the background of resolved or unresolved stars and gaseous emission at any wavelength. A detailed description of this technique is given in Roth et al. (2003). Application of image deconvolution techniques is able to disentangle the different components from severely crowded stellar fields with significant contamination from nebular emission. We used the cplucy two-channel algorithm, which is available in IRAF. A more detailed account of this technique is given in Becker (2002) and Becker et al. (2003).

In Fig. 1 we present continuum maps of B 416 from a wavelength region near $6000 \AA$ of MPFS and INTEGRAL, respectively. For each IFU map the same FOV as observed with HST $^{3}$ is shown for comparison. While the MPFS map (a) represents the intensity distribution exactly over the square spaxels of the lens array, the INTEGRAL map (c) was interpolated from the 205 data points of the fiber bundle (overlay). Note the patches of low intensity near the upper left corner in (c) indicating the presence of dead fibers in the bundle.

While the MPFS observations suffered from rather poor seeing, the conditions with INTEGRAL were more favourable

\footnotetext{
${ }^{3}$ Based on observations made with the NASA/ESA Hubble Space Telescope, obtained from the data archive at the Space Telescope Science Institute. STScI is operated by the Association of Universities for Research in Astronomy, Inc. under NASA contract NAS 5-26555.
}

and yielded much better spatial resolution. In Fig. 1a the image of B 416 is blended with the star cluster located from 2.. 5 to 5.'5 to the SW. In Fig. 1c the cluster is clearly separated from B 416 and marginally resolved into stars. Three other isolated stars seen in the HST frame to the W of B 416 are also marginally present in the UNTEGRAL map.

\section{Results}

\subsection{The nebula around $B 416$}

Figure 2 presents monochromatic images of B 416 in the emission lines of $\mathrm{H} \alpha, \mathrm{H} \beta, \mathrm{He} \mathrm{I} \lambda 5876$, [SII] $\lambda 6717+\lambda 6731$, [NII] $\lambda 6583$, and [OIII] $\lambda 5007$ from the MPFS observations. The images were produced in $\pm 2.5 \AA$ bands from the central pixels of the lines $(7.5 \AA$ bands) as relative fluxes. Corresponding continuum images were substracted. There is a shift in the monochomatic pictures depending on wavelength due to atmospheric dispersion. Its value is 0 '. $^{\prime} 42 / 1000 \AA$ ( $0 . ' 65$ between $\mathrm{H} \alpha$ and [OIII] $\lambda 5007)$ towards the NW with decreasing wavelength. The central star position is indicated in the figure by crosses in each image taking the atmospheric shifts into account.

A ring-like nebula is clearly seen in the emission line maps of Fig. 2. It is non-symmetrical and presents a different morphology in lines of different excitation. The star itself is a source of emission in permitted lines. The stellar emission in $\mathrm{H} \alpha$ is particularly strong and gives rise to a high level of contrast, which is why the nebula appears faint at this wavelength. There is no apparent forbidden line emission coincident with the PSF of the central star. The inner parts of the nebula are filled with faint and diffuse emission in [OIII] $\lambda 4959,5007$.

In order to disentangle nebula and stellar PSF components in the spectral domain we attempted a gaussian decomposition of the nebular and stellar emission line profiles in $\mathrm{H} \alpha$ and $\mathrm{H} \beta$. Since the nebular line is intrinsically narrow, but the stellar emission line profile is expected to be broader under the influence of a strong stellar wind, this approach seemed to provide a useful criterion.

As a best guess we estimated the width of the nebular emission in $\mathrm{H} \alpha$ and [NII] $\lambda 6548,6584$ in regions far away from the star, yielding a gaussian $F W H M \approx 6.8 \AA$, which is in fact identical to the spectral resolution. We then fitted the observed spectrum near $\mathrm{H} \alpha$ by three gaussians for $\mathrm{H} \alpha$ and [NII] $\lambda 6548,6584$, whose widths are equal to that of the instrumental profile, and by a fourth component for the stellar $\mathrm{H} \alpha$ emission, whose $F W H M$ was determined from the fit. In fact, for all spaxels close to the centroid of the star, this latter component makes a non-negligible contribution to the spectrum and has an $F W H M \approx 12.2 \AA$. The broad component contributes to $55 \%$ of the total $\mathrm{H} \alpha$ emission inside of the centroid of the star, but it is absent in spaxels far away from the star. The observed spectrum in a spaxel close to the star, the three narrow and one broad gaussian fits, and the sum of the fits are plotted in Fig. 3. This spectrum was extracted in a spaxel not far from the star to show both broad and narrow components of $\mathrm{H} \alpha$. The flux units are the same as in Fig. 2, whose images were produced in $7.5 \AA$ bands. Similarily, the decomposition of 
$H \alpha$

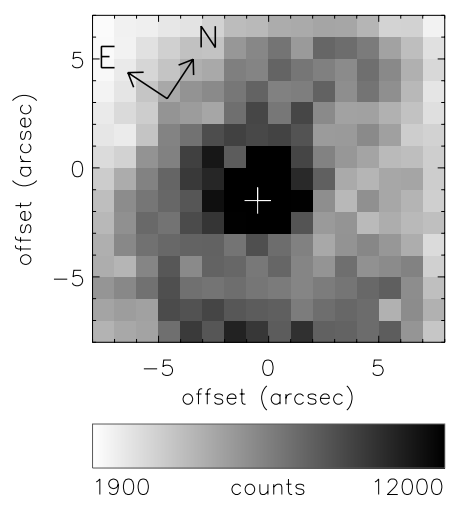

[SII] $6717+6731$

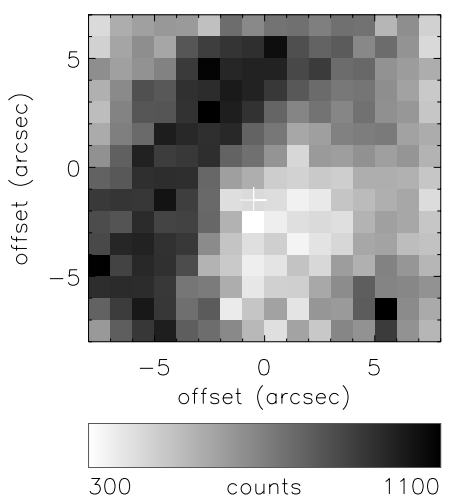

$H \beta$

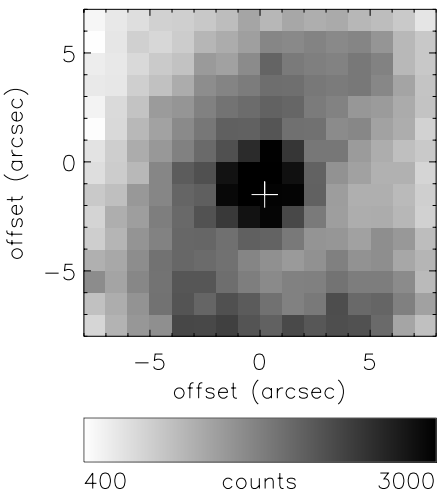

[NII] 6583

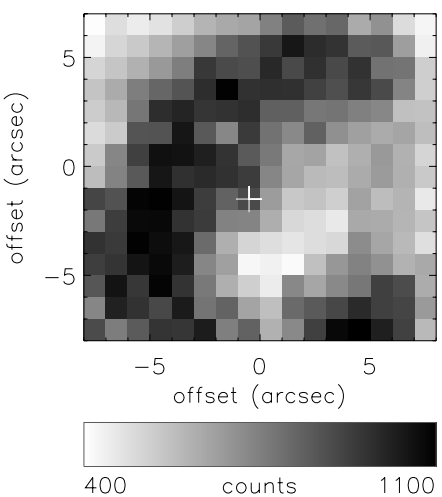

Hel 5876

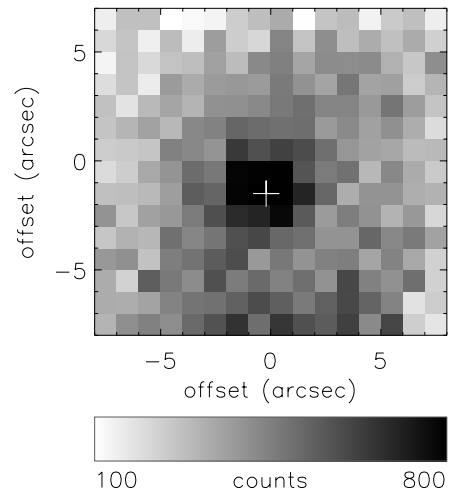

[OIII] 5007

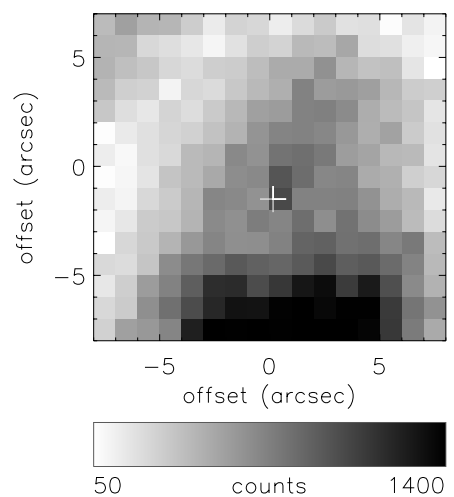

Fig. 2. Monochromatic MPFS maps of B 416 taken in central $7.5 \AA$ A bands of different emission lines, continuum subtracted. Intervals of relative fluxes (counts) used in the maps are shown. The position of the star is indicated by a white cross. A prominent arc-like nebular feature is visible to the left. The star itself shows emission in the Balmer lines and He I.

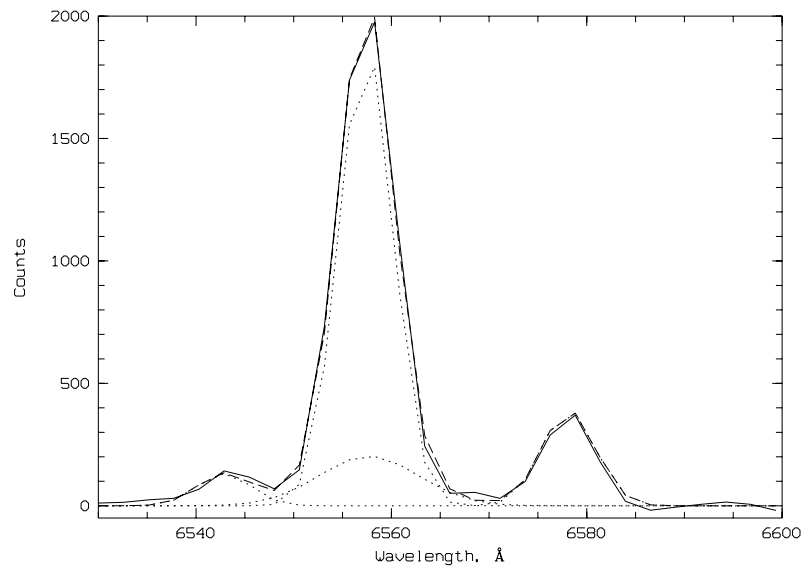

Fig. 3. An example of the $\mathrm{H} \alpha$ and $[\mathrm{NII}] \lambda 6548,6584$ lines (solid line) in a region close to the star. They are fitted with four gaussian profiles (dashed lines) - broad and narrow $\mathrm{H} \alpha$ lines and two narrow [NII] lines. A sum of the gaussian profiles is shown by a long-dashed line.

$\mathrm{H} \beta$ into broad and narrow profiles resulted in a broad line width of $F W H M \approx 10 \AA$. This value agrees well with the value found in $\mathrm{H} \alpha$ and with the corresponding intrinsic velocities.

Figure 4 demonstrates a spatial representation of the spectral decomposition into narrow and broad profiles, where the broad component is associated with the star and is perfectly coincident with the stellar PSF. In the nebular analysis, which follows below, we only use the narrow Balmer line component. These results suggest that the broad Balmer emission lines are indeed most likely formed in the atmosphere of the star. We must stress both that an expanding nebula near the star (with an angular extent below our spatial resolution) cannot entirely be ruled out, and that even the stellar atmosphere can contribute to the narrow emission.

The morphology of the ring-like nebula presents an almost perfect elliptical ring (Fig. 2), which is however interrupted on its NW side by a distinct gap. The overall surface brightness distributions in $\mathrm{H} \alpha$, [NII], and [SII] are fairly similar, but show differences in the exact size and morphological details. The surface brightness distribution in [OIII] nebula is strikingly different from those in the $\mathrm{H} \alpha$, [SII], and [NII] lines. The extended faint emission in the $\mathrm{N}, \mathrm{W}$ parts and very bright $\mathrm{SW}$ regions are dominated by high excitation gas contrary to those parts of opposite directions. The $\mathrm{H} \beta$ nebula pattern (and $\mathrm{H} \gamma$, which is not shown here) is somewhere intermediate between those of high excitation ([OIII]) and low excitation ([SII] and [NII]).

To illustrate the whole nebula around B 416, we present in Fig. 5 a small (roughly 1 arcmin) subfield from $\mathrm{H} \alpha$ and [OIII] images of M 33 taken by Massey et al. (2001) in the course of an imaging survey of Local Group galaxies, though the $\mathrm{H} \alpha$ nebula was first detected by Shemmer et al. (2000). The images of 

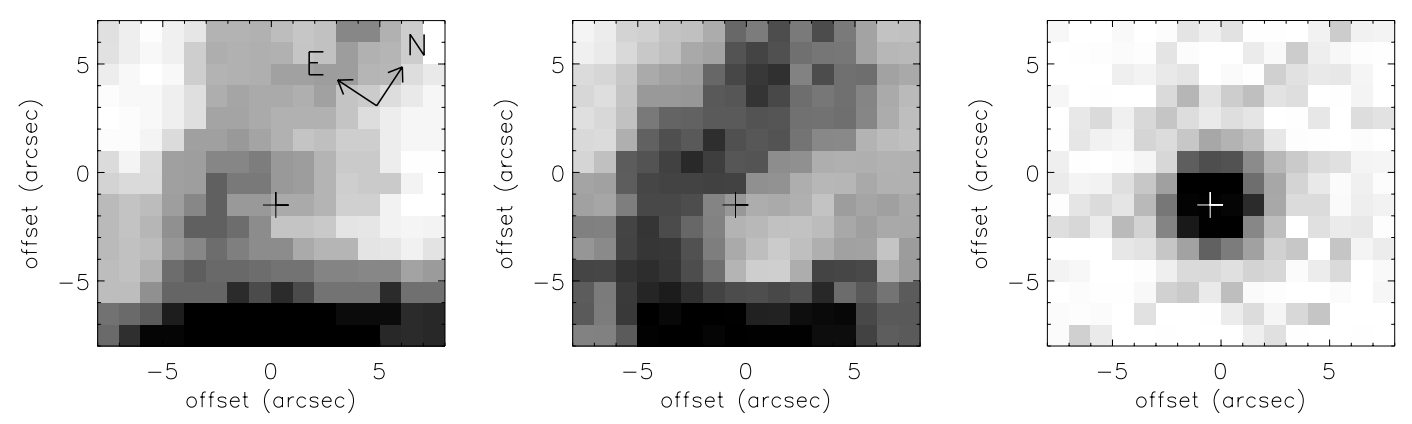

Fig. 4. Maps of $\mathrm{B} 416$ : narrow $\mathrm{H} \beta$ component (left panel), narrow $\mathrm{H} \alpha$ (middle), and broad $\mathrm{H} \alpha$ (right). The stellar centroid is indicated by a cross in each frame.

Massey et al. (2001) were obtained with the CTIO and KPNO 4-m telescopes using the Mosaic CCD cameras. Narrow-band filters were used with bandwidths of $55 \AA$ in $[\mathrm{OIII}]$ and $80 \AA$ in $\mathrm{H} \alpha$. The MPFS field and corresponding emission line isophotes of the narrow $\mathrm{H} \alpha$ line and [OIII] $\lambda 5007$ are overplotted. Each step in the isophotes corresponds to 200 counts, the same relative units as used in Fig. 3. The maximum isophote level is 1900 counts in $\mathrm{H} \alpha$ and 900 counts in [OIII] 25007 . The MPFS maps are in excellent agreement with these direct images.

The [OIII] emission around B 416 is faint. It is concentrated on the SW side of the nebula, where an extended [OIII] region does exist, which is only partly covered by the MPFS field. The most distant SW part of the nebula (just outside of the MPFS map) has about the same shape in [OIII] and $\mathrm{H} \alpha$ (Fig. 5). This fact, together with the specific shape of the [OIII] nebula, may point towards an explanation that the most distant SW part of the whole nebula is shaped not only under the influence of B 416, but also by a cluster of hot stars. Two compact clusters of stars (Fig. 1b) are located in these regions, coinciding with the [OIII] bright emission of the nebula, where, conversely, [SII] and [NII] are relatively faint. Yet another high excitation knot is seen within the diffuse [OIII] region, just $3^{\prime \prime} \mathrm{SW}$ of the edge of the MPFS FOV (Fig. 5). One could speculate that the bright [OIII] emission is due to photoionization from hot stars in these clusters, whose relation, however, to the ring-like nebula is not immediately clear.

A similar situation is observed in the nebula DEM L106 around LMC B[e]-supergiant Hen S22 (Chu et al. 2003). The $\mathrm{H}$ II region N30B with a cluster of hot stars is located inside the large nebula ( $\sim 30 \mathrm{pc}$ in radius). A bow-shock-like halo around $\mathrm{N} 30 \mathrm{~B}$ is oriented towards Hen S22. It is formed as a result of interaction with the stellar wind of Hen S22. The halo reflects the supergiant radiation. One could conclude that the most distant SW region of the ring-like nebula of B 416 is probably affected in a similar fashion in the environment of the hot star cluster.

For a qualitative analysis of the excitation structure of the nebula we present "ionization" maps of B 416 in Fig. 6. Low excitation regions are traced by the $[\mathrm{SII}] / \mathrm{H} \alpha$ map, whereas high excitation regions appear in the $[\mathrm{OIII}] / \mathrm{H} \beta$ ratio map. In both cases we used the Balmer line maps derived from the narrow line components as described above. One can see from a comparison of Figs. 6, 2, and 4 that over a wide range of position angles, the inner parts of the ring-like nebula are dominated by high excitation, as opposed to the outer rim of the ring, which is most pronounced in the low ionization radiation of [SII]. This observation is qualitatively in line with the expectation of finding (i) photoionization predominantly near the star and (ii) shock excitation from the interaction of the expanding shell with the interstellar medium at the edge of the nebula.

We measured the diagnostic line ratios of $\mathrm{H} \alpha /[\mathrm{NII}]$, $\mathrm{H} \alpha /[\mathrm{SII}]$ (sum the doublets) and $I(6717) / I(6731)$ for [SII], and plotted the results in the diagnostic diagrams of Sabbadin \& D'Odorico (1976). Our mean values for the nebula around B 416 are as follows: $\mathrm{H} \alpha /[\mathrm{NII}]=3.24 \pm 0.39, \mathrm{H} \alpha /[\mathrm{SII}]=$ $2.21 \pm 0.25$, and $I(6717) / I(6731)=1.61 \pm 0.11$, where the r.m.s. values are for individual resolution elements. The mean line ratios are quite typical for HII regions; however, the ratios are not constant accross the nebula. It is directly seen in Fig. 2 that the rim is more distant in [SII] than in [NII] and $\mathrm{H} \alpha$. The outer parts of the ring, at least in the NES directions, consist of a gas of lower excitation. This conclusion is confirmed by the $[\mathrm{SII}] / \mathrm{H} \alpha$ ratio map (Fig. 6). The ratio is greater than 0.4 in the outer parts of the ring, which indicates a collisional excitation of gas as was confirmed by Smith et al. (1993) for SNRs in M 33. The electron density (Osterbrock 1989) over the whole nebula was found to be at the low density limit, $n_{\mathrm{e}}<100 \mathrm{~cm}^{-3}$, with an average value of $n_{\mathrm{e}} \sim 10 \mathrm{~cm}^{-3}$.

The lower left panel of Fig. 6 shows a map of the $\mathrm{H} \alpha / \mathrm{H} \beta$ ratio, whose canonical value based on recombination theory is expected to be $\approx 3$. A systematic increase of this value is seen along the $\mathrm{E}$ rim of the ring-like nebula. Most strikingly, however, the ratio increases to a value of 5 at the $\mathrm{N}$ border of the map. This peculiar region happens to coincide with the aforementioned gap in the structure of the ring (Fig. 5), which is thus explained as extinction from dust.

A point-by-point analysis of radial velocities in $\mathrm{H} \alpha$, [OIII] $\lambda 5007$, and [SII] $\lambda 6117$ lines was performed by fitting gaussians to each line. The internal accuracy of the instrumental wavelength calibration was increased by measuring differentially against the [OI] $\lambda 5577$ and $\lambda 6300$ night sky lines. The resulting accuracy of the radial velocity measurements is about $10 \mathrm{~km} \mathrm{~s}^{-1}$ per spatial element $\left(1^{\prime \prime}\right)$ in those places where the nebular emission provides sufficient intensity (Fig. 2). The final radial velocity maps were averaged over 3 pixels, and the resulting radial velocity accuracy is better than $10 \mathrm{~km} \mathrm{~s}^{-1}$ per resolution element.

The lower right panel of Fig. 6 presents a radial velocity map as derived from the deblended [SII] $\lambda 6717$ emission line. 

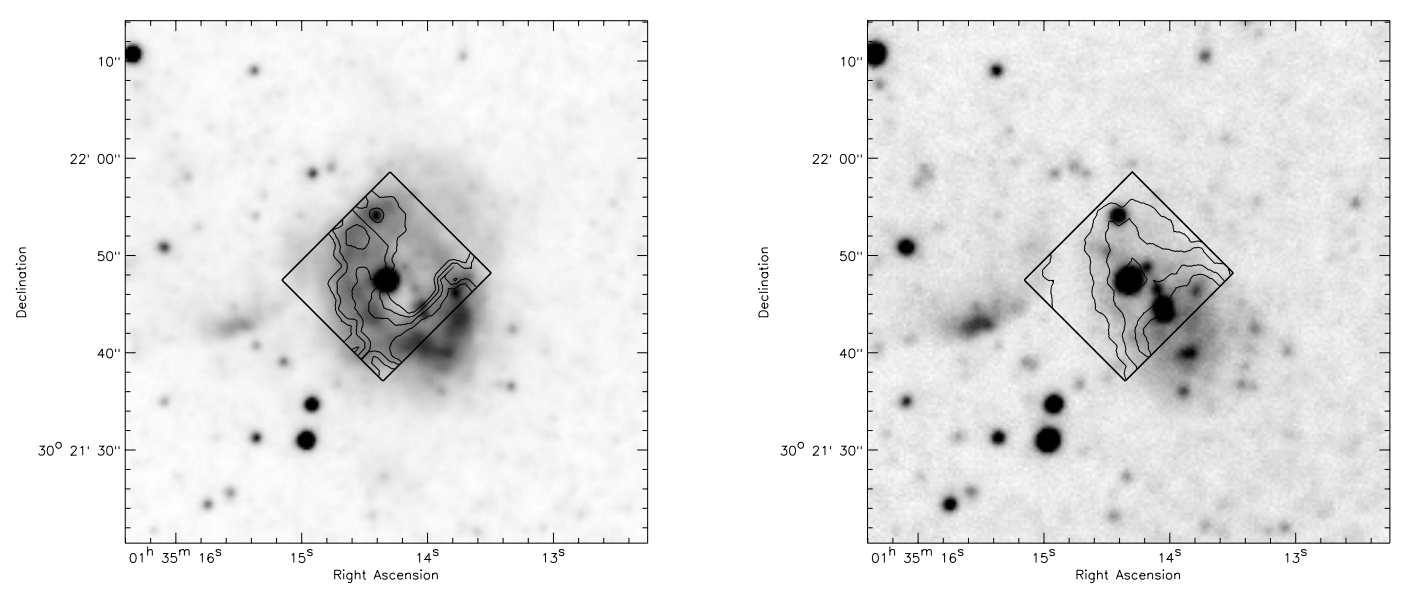

Fig. 5. $\mathrm{H} \alpha$ (left) and [OIII] $\lambda 5007$ (right) images of the B 416 region taken by Massey et al. (2001) with the MPFS field and the line isophotes imposed on the images.
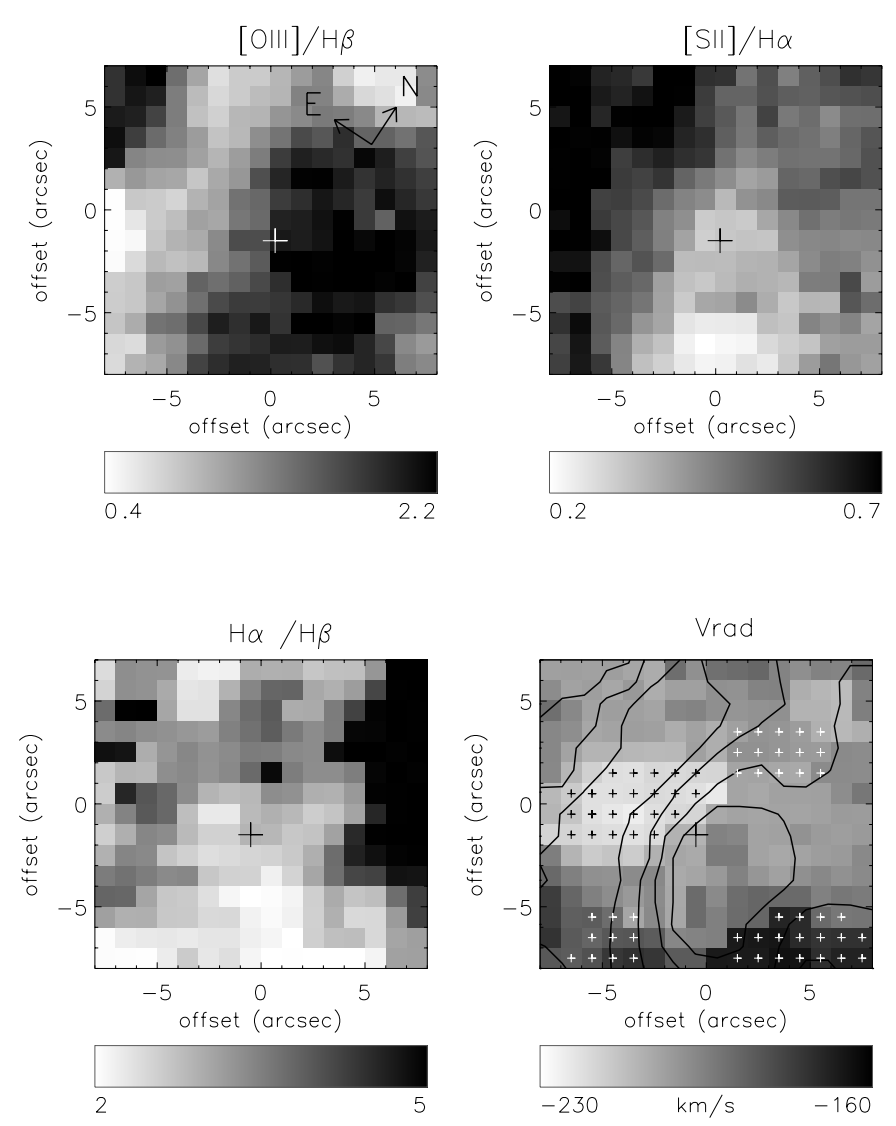

Fig. 6. Ionization maps of B 416 obtained as ratios of line intensities of [OIII] $\lambda 5007 / \mathrm{H} \beta$ (narrow), [SII] $(\lambda 6717+\lambda 6731) / \mathrm{H} \alpha$ (narrow), a map of $\mathrm{H} \alpha / \mathrm{H} \beta$ ratio and radial velocity map obtained in [SII] $\lambda 6717$ emission with relative flux isophotes of the sum of the lines of the [SII] doublet (compare with Fig. 2). Crosses indicate 4 selected regions where mean radial velocities were measured (see text).

The overplotted isophotes were derived from the total intensity of the doublet [SII] $\lambda \lambda 6717,6731$ (cf. Fig. 2). We chose this emission line because it is bright and ubiquitous over the entire FOV, thus minimizing the uncertainty of our measurement. The overall velocity field pattern, however, is the same for all of the bright nebular lines, and shows a systematic variation with a peak-to-peak amplitude of about $30-40 \mathrm{~km} \mathrm{~s}^{-1}$. There are two conspicuous features of extreme radial velocities: an area of roughly $9^{\prime \prime} \times 5^{\prime \prime}$ across the E rim of the nebula has a minimal velocity of $\approx-226 \mathrm{~km} \mathrm{~s}^{-1}$, whereas an area of the same extension and at the same distance to the $\mathrm{W}$ of the star has a significantly more positive radial velocity of $\approx-184 \mathrm{~km} \mathrm{~s}^{-1}$. In the N-S direction perpendicular to these two patches the radial velocity is constant $\left(\Delta V_{r}<5 \mathrm{~km} \mathrm{~s}^{-1}\right)$ and zero with respect to the average, except for the $\mathrm{S}$ corner of the field, where the velocity is about $+10 \mathrm{~km} \mathrm{~s}^{-1}$ more positive than the average.

We derived mean radial velocities of $\mathrm{H} \alpha$, [SII] $\lambda 6717$, and [OIII] $\lambda 5007$ lines in four selected $\mathrm{E}, \mathrm{N}, \mathrm{W}$, and $\mathrm{S}$ regions marked by crosses in Fig. 6. The mean velocities of the $\mathrm{H} \alpha$, [SII], and [OIII] lines respectively are in the E-region $-239 \pm 3,-226 \pm 2,-246 \pm 8 \mathrm{~km} \mathrm{~s}^{-1}$; in N-region $-226 \pm 2$, $-210 \pm 3,-232 \pm 5 \mathrm{~km} \mathrm{~s}^{-1}$; in W-region $-212 \pm 3,-184 \pm 4$, $-216 \pm 3 \mathrm{~km} \mathrm{~s}^{-1}$, and in S-region $-220 \pm 3,-197 \pm 7,-213 \pm$ $4 \mathrm{~km} \mathrm{~s}^{-1}$. The errors of the mean values depend both on brightness and homogeneity of a line emission in these selected regions. Some systematic differences in radial velocities between these three lines may appear because they were formed under different physical conditions (unresolved gas clouds and structures). The measurements in the selected regions confirm the total radial velocity gradient observed in the nebula in the E-W direction. It is $\Delta V_{r}(E W)=33 \pm 8 \mathrm{~km} \mathrm{~s}^{-1}$ over these three lines, and is $\Delta V_{r}(E W)=34 \pm 4 \mathrm{~km} \mathrm{~s}^{-1}$ in $\mathrm{H} \alpha+$ [SII] $\lambda 6717$, which are the most similar both in physical conditions and in nebula patterns (Fig. 2).

The star's radial velocity in $\mathrm{H} \alpha$ (the total profile) is $-231.1 \pm 3.5 \mathrm{~km} \mathrm{~s}^{-1}$. It is intermediate between the $\mathrm{E}$ and $\mathrm{W}$ opposite features of extreme radial velocities in $\mathrm{H} \alpha$ and closer to radial velocity of the $\mathrm{E}$ region in accordance with location of the star inside the ring-like nebula (Fig. 2). The stellar radial velocity may be considered as the systemic velocity of the whole complex (star + nebula). However the total stellar $\mathrm{H} \alpha$ line profile may be distorted because of the contribution $(50 \%)$ by the broad stellar component and stellar wind.

We summarize our findings that the morphological asymmetry of the nebula in the E-W direction is accompanied by an asymmetry in the radial velocity pattern: the E part of the 

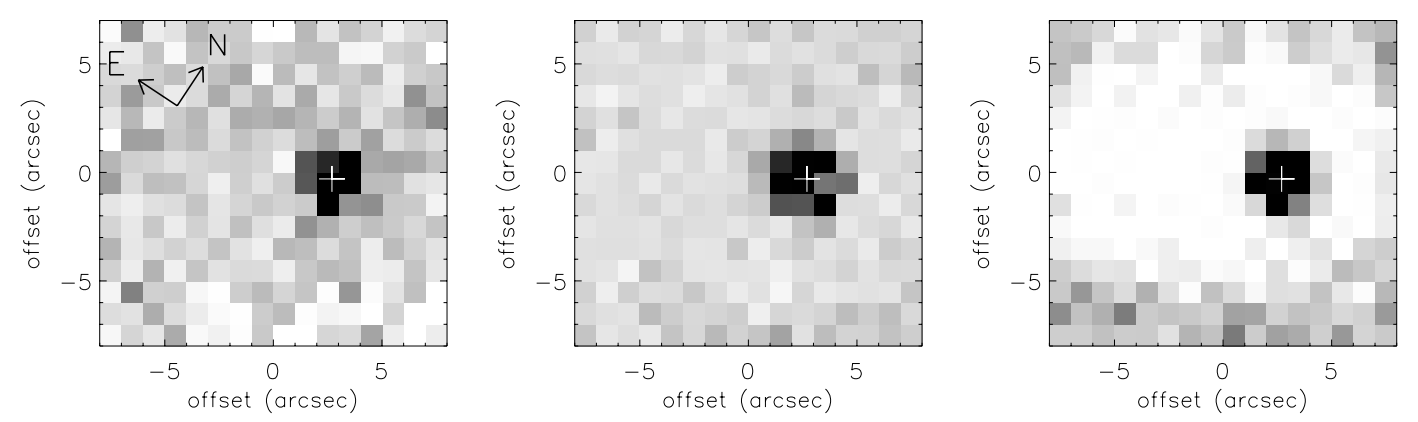

Fig. 7. v 532 maps in broad $\mathrm{H} \beta$ (left), narrow $\mathrm{H} \beta$ (middle), and in the nearby continuum (right panel). Crosses mark the centroid of the star.

ring approaches $\left(\approx-15 \mathrm{~km} \mathrm{~s}^{-1}\right)$, while the $\mathrm{W}$ part receedes $\left(+15 \div+20 \mathrm{~km} \mathrm{~s}^{-1}\right)$. There is no apparent asymmetry in the $\mathrm{N}-\mathrm{S}$ direction. The asymmetry of the nebula in the $\mathrm{E}-\mathrm{W}$ direction is accompanied also by an asymmetry in the gas excitation, while the approaching $\mathrm{E}$ part of the ring is dominated by low excitation emission (a collisional excitation in the rim), but the receeding $\mathrm{W}$ part is dominated by high excitation gas (a radiative excitation by the central star).

\subsection{A nebula around v532 (Romano's star)}

Our MPFS images of v 532, another LBV-candidate in M 33, were taken merely in the spectral range 4470-5800 $\AA$ (spectral resolution $\approx 3.5 \AA$ ). In the absence of $\mathrm{H} \alpha$ and [SII] we had to resort to an analysis in $\mathrm{H} \beta$, since no [OIII] $\lambda 4959,5007$ were detected in the spectrum. Although at first glance the $\mathrm{H} \beta$ and continuum maps of this object appeared point-like, a suspicious slight elongation of the stellar image in $\mathrm{H} \beta$ with $\mathrm{PA} \sim 35^{\circ}$ led us to perform a gaussian decomposition of the spectral profile into a broad and a narrow component along the lines of the exercise with B 416 as described above. As before, we stress that besides an extended nebula, a narrow component may either originate in the stellar atmosphere or in a nearby unresolved nebula, or in both. We obtained a good fit from two gaussians with $F W H M($ narrow $)=4.1 \AA$ and $F W H M($ broad $)=11.1 \AA$.

The resulting maps of $\mathrm{v} 532$ in broad $\mathrm{H} \beta$, narrow $\mathrm{H} \beta$, and in the nearby continuum are shown in Fig. 7. Note that the narrow-line component is a factor of 4 brighter than the broad component. In order to assess the possible spatial extent of these surface brightness distributions, which are close to unresolved point sources, we performed a Moffat fit to the three maps and inspected their radial intensity profiles for comparison. Figure 8 shows that the narrow component is more extended (3". $1 F W H M)$ than the broad component (2'. 6 FWHM), which in turn has the same spatial extent as the continuum intensity profile. The difference between the broad and narrow component intensity profiles becomes significant at a scale greater than the seeing value, $\approx 2^{\prime \prime} F W H M$. The error bars in the intensity profiles are about the same. In five consequent bins from $3^{\prime \prime}$ to $5^{\prime \prime}$ the difference between the broad and narrow component energy distributions varies from 3.5 to 2.5 standard deviations. We conclude that an extended nebula has been marginally detected in the narrow emission line component of the object.

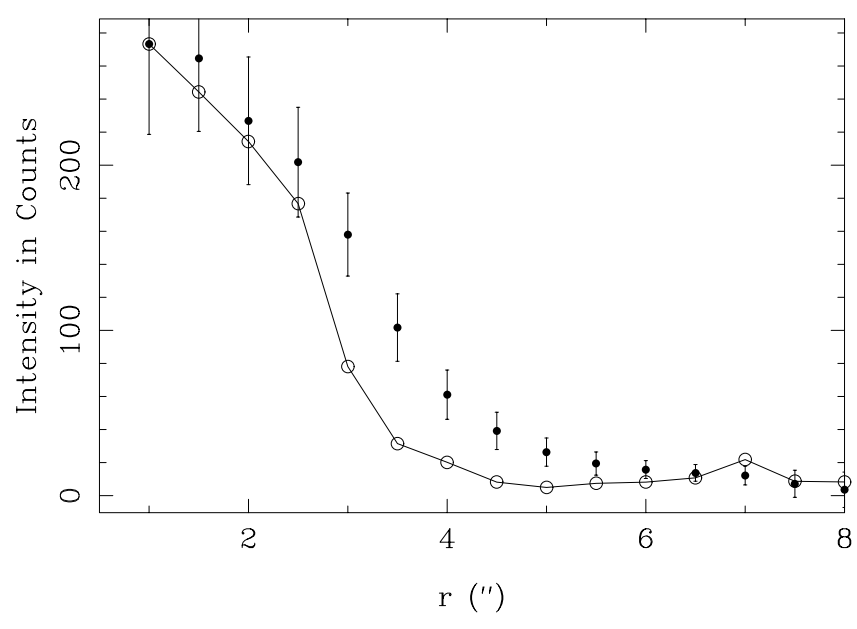

Fig. 8. Radial intensity profiles of v 532 maps in narrow (filled symbols) and broad components (open circles) of the $\mathrm{H} \beta$ line profile.

A radial velocity analysis of the narrow $\mathrm{H} \beta$ component supports this view. We measured the radial velocity in each spaxel differentially against the night sky [OI] $\lambda 5577$ emission line as a reference. The central part including the star has a velocity $\approx-220 \mathrm{~km} \mathrm{~s}^{-1}$. The SW part of the nebula shows on average positive relative velocities, while the velocities of the NE part are mainly negative. In order to check this finding against any potential errors from the spectral decomposition, we repeated the velocity measurements by fitting single gaussian profiles to the spectra which had been coadded from several spaxels in three representative regions (Fig. 9): center (12 spaxels), NE (9 spaxels), and SW (4 spaxels). This procedure increases the $\mathrm{S} / \mathrm{N}$ ratio in the resulting compound spaxels. The derived $\mathrm{H} \beta$ radial velocities are $-219 \pm 2.7 \mathrm{~km} \mathrm{~s}^{-1}$ in the central part, $-191 \pm 10 \mathrm{~km} \mathrm{~s}^{-1}$ in the $\mathrm{SW}$, and $-235 \pm 11 \mathrm{~km} \mathrm{~s}^{-1}$ in the NE part.

The analysis confirms the presence of a radial velocity gradient across the nebula; the SW side of the nebula recedes and the NE side approaches. The total radial velocity gradient amounts to $44 \pm 11 \mathrm{~km} \mathrm{~s}^{-1}$. The total angular size of the nebula is $\approx 9^{\prime \prime}(\approx 30 \mathrm{pc}$ in projection $)$ in the NE-SW direction. From the heterogeneous velocity distribution in Fig. 9 one may suspect that the system is more complex than just the simple picture of a bipolar nebula with receding and approaching lobes. Given the limited spatial resolution of our data we therefor forgo any further analysis. 


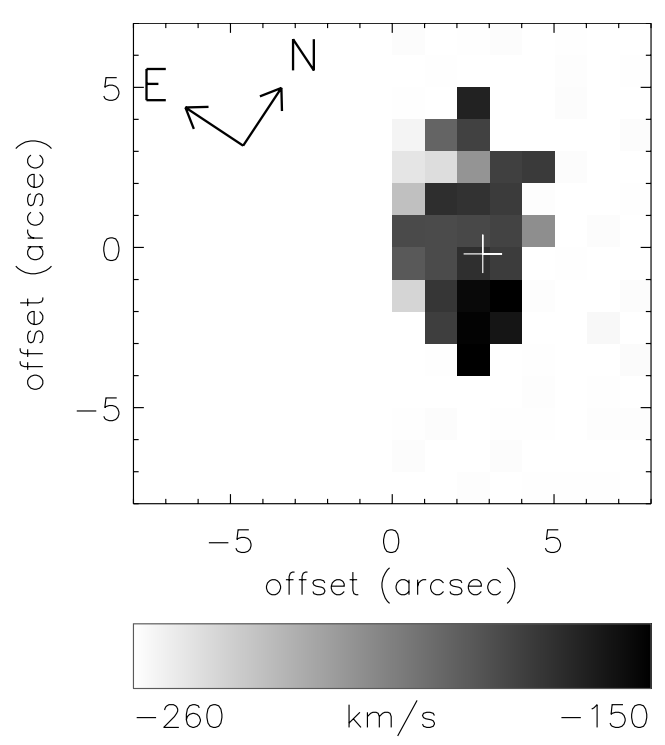

Fig. 9. v 532 nebula radial velocity map derived in $\mathrm{H} \beta$ line. The cross marks the position of the star.

\subsection{The spectra of $B 416$ and $v 532$}

3D and longslit spectra of B416 and v532 are shown in Fig. 10. Three rectified spectra of B 416 from different instruments are plotted with an offset for clarity. The top spectrum was obtained with the BTA long-slit spectrograph on January 19, 2001 (a spectral resolution $F W H M \approx 7 \AA$ ). The middle spectrum was obtained by coadding spaxels with a digital aperture of the MPFS datacube, observed on September $28,1998(F W H M \approx 7 \AA)$. The bottom spectrum comes from the INTEGRAL datacube, which was observed on January 18 , $2001(F W H M \approx 11 \AA)$. The lower panel in Fig. 10 presents two v 532 spectra: the top spectrum was obtained with MPFS on September 18, $1998(F W H M \approx 3.5 \AA)$ and the lower spectrum from the long-slit spectrograph, observed on July 12, 1999 $(F W H M \approx 3.5 \AA)$.

A comparison of the long-slit (LS) B 416 spectrum with its 3D spectroscopy counterparts (MPFS, INTEGRAL) demonstrates, that the former is severely affected by nebular contamination, e.g. [OIII] $\lambda 4959,5007$. The strongest line [OIII] $\lambda 5007$ is superposed on a blend of Fe II emission lines in B 416 spectrum. The nebular lines, however, are absent in the MPFS and INTEGRAL plots, because the process of 2-dimensional background correction accurately subtracts the local contribution of this light. In general, the flux distribution along a slit is not really adequate to estimate the background at the location of the object.

We inspected the spectra for spectral variability. According to Shemmer et al. (2000), B 416 shows only weak photometric and spectral variability, which is more or less in line with our observations taken essentially at two epochs in 1998 and 2001 (the LS and INTEGRAL spectra were coincidently taken only one night apart from each other). Considering the difference in spectral resolution, the LS spectra are indistinguishable from INTEGRAL, despite the systematic [OIII] contamination at $\lambda \lambda 4959,5007$. There are some small differences between LS and MPFS spectra. The situation is quite different in the case
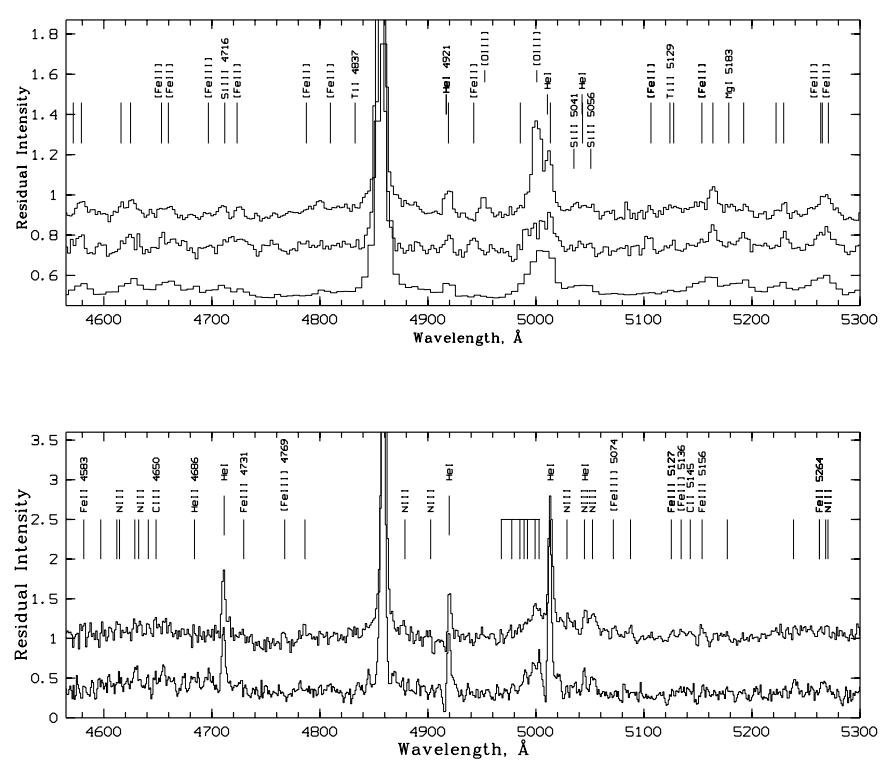

Fig. 10. Normalized spectra of B 416 (top panel), and v 532 (bottom panel). B 416 spectra are from INTEGRAL (bottom), MPFS (mid$d l e$ ), and the LS-spectrograph (top). v 532 spectra are from the LSspectrograph (bottom) and MPFS (top). Numerous FeII lines in the B 416 spectrum and NII lines in v 532 spectrum are not labeled in order not to confuse the plot.

of v 532, which shows a pronounced onset of P Cyg profiles in He I between 1998 (MPFS) and 1999 (LS).

The most prominent lines in both stars are the strong emission hydrogen lines with broad wings and narrower He I lines. He I $\lambda 4922,5015$ lines in B 416 are blended with the brighter Fe II $\lambda 4923,5018$ lines, but the single He I $\lambda 5876,6678$ lines are clearly present in the red part.

The main difference in the spectra of these stars consists, however, in the chemical composition of their atmospheres. There are many Fe II lines in B 416 and N II lines in v 532. In B 416 besides Fe II, Fe III, and He I lines, the following ions are also detected: Si II, Cr II, N II, Ti II, S II, C II, and also neutral elements such as $\mathrm{TiI}$ and $\mathrm{Mg}$ I are present in the atmosphere. The Fe II wavelengths are indicated in the B 416 spectrum (unlabeled in Fig. 10), while the same is true for some N II lines in the v 532 spectrum.

The star v 532 exhibits appreciable spectral and photometrical variability (Fabrika 2000; Kurtev et al. 2001; Polcaro et al. 2003). In some epochs strong P Cyg components appear in $\mathrm{He} \mathrm{I}$ or the hydrogen lines. Sometimes the He I emission component disappears, sometimes it becomes very bright, and the He II $\lambda 4686$ line appears in emission. In our MPFS spectrum the He I lines are bright with weak blue-shifted absorption components. A P Cyg line profile is more clearly seen in He I $\lambda 4922$, split by $220 \mathrm{~km} \mathrm{~s}^{-1}$. A faint He II $\lambda 4686$ line is possibly present (Fig. 10) with P Cyg-like profile. The spectrum is strongly blended by weak emission lines of N II, N III, Fe II, Fe III, C II, C III, Si II, Si III. Faint emissions of Ti, Cr, and S are also observed. The most numerical are nitrogen lines. A typical N II broad emission blend $\approx 5000 \AA$ is observed. The signal-to-noise value in the MPFS spectrum is at least 30 in 
$4700 \AA$ and $5300 \AA$ regions, and is no less than 40 around $5000 \AA$ A.

Both stars possess extended atmospheres, as is apparent from the presence of the forbidden [FeIII] and [FeII] lines, the brightest of which are marked in the spectrum. We have also detected the [NII] $\lambda 5754.8$ forbidden line in both stars (not shown in Fig. 10).

\section{Discussion and conclusions}

One may suggest that B 416 is surrounded by an expanding ring-like nebula, whose overall morphological, kinematic, and gas excitation properties are compatible with an outflow from the star, which happened to be confined to a plane. If the ringlike appearance were due to an increased column-density near the edge of an assumed spheroidal nebula, we would not expect to find the two patches of extreme radial velocities confined to the approaching and receding parts of the ring. It is possible that the plane of the torus is coincident with the equatorial plane of the star. The interior region of the nebula is filled with hot, high excitation gas. The pronounced low excitation emission near the edge of the ring indicates a shock ionization of material running into the interstellar medium.

Fitting an ellipse to the entire $\mathrm{H}_{\alpha}$ images of Massey et al. (2001) in Fig. 5 and of Shemmer et al. (2000) and assuming an intrinsic circular symmetry of the ring, we estimate the ring axis inclination to the line of sight as $i \sim 30^{\circ}$. From the apparent radial velocity gradient of $\approx 20 \mathrm{~km} \mathrm{~s}^{-1}$, we roughly estimate the deprojected physical expansion velocity as $\sim 40 \mathrm{~km} \mathrm{~s}^{-1}$. The physical dimension of the nebula is measured from the major axis along the $\mathrm{N}-\mathrm{S}$ direction as a radius of $\approx 30 \mathrm{pc}\left(9^{\prime \prime}\right)$. From these numbers we find a dynamical lifetime of the nebula of $t_{\mathrm{d}} \sim 8 \times 10^{5}$ years. This estimate indicates a main-sequence or a pre-LBV status of the star. The nebula is probably a residual MS bubble. Using the average density of the nebula found above $\left(n_{\mathrm{e}} \sim 10 \mathrm{~cm}^{-3}\right)$, we estimate that a total mass of the nebula is about a few thousand solar masses. Such a big nebula may be composed mainly of swept up interstellar gas.

The photometry of this star has been studied well (Shemmer et al. 2000), but it does not show the variability that is typical of LBVs. Using the Sternberg Institute plate collection, Zharova (2004) has found that for the last 50 years a variability of B 416 was notably less than $1 \mathrm{mag}$. There are instead minor long-term (1987-1997) variations of about 0.15 (Shemmer et al. 2000).

Spectrally, B 416 has to be classified as an "iron-star" (Walborn \& Fitzpatrick 2000). It is the brightest supergiant in M 33 with UV-excess (Massey et al. 1996). Based on spectral criteria, luminosity, and the absence of a large-amplitude variability, B 416 may be classified as B[e]-supergiant star (Zickgraf et al. 1986; Lamers et al. 1998b). The spectrum is typical of [Be]-supergiants (or "sg[Be]"stars, Lamers et al. 1998b) - very bright hydrogen lines, a forest of narrower Fe II lines, He I lines, forbidden lines.

The presence of anisotropical mass loss is a common and distinguishing property of $\mathrm{B}[\mathrm{e}]$-supergiants (Lamers et al. 1998b). UV resonance line profiles in the edge-on inclination stars show that the stellar winds are very slow in massive
$\mathrm{B}[\mathrm{e}]$-supergiants (Zickgraf 2003). The expansion velocities are $\sim 70-100 \mathrm{~km} \mathrm{~s}^{-1}$, a factor of 10 less than usually observed in stars of a similar spectral type. Such velocities are also typical of disk-like outflows in close binaries, where a donor star overfills its critical Roche lobe. Binaries among B[e]-supergiants were reviewed by Zickgraf (2003), while Sholukhova et al. (2004) have studied radial velocities in B 416 and found the periodical variability with a period $\sim 16$ days. We postpone a discussion of B 416 as a close binary for a future paper.

The second star of our study, v 532 shows a typical LBVlike variability (Romano 1978; Kurtev et al. 2001; Sholukhova et al. 2002). Over the past 50 years it has changed its brightness over a range of $B=16.3 \div 18.4$. There are large amplitude variations with a time-scale of years, onto which a sporadic $0 \mathrm{~m} \cdot 3-0 \mathrm{~m} \cdot 5$ amplitude variability is superimposed with a time-scale of several months. The last irregular minimum occurred from 1974 to 1981, and the last (also quite irregular) maximum was observed on 1990-1995. At the time of our spectroscopic observations in September 1998 the star had intermediate, gradually decreasing brightness. We estimate the brightness of $\mathrm{v} 532$ during this epoch as $B=17.5 \pm 0.15$ (Sholukhova et al. 2002).

During an intermediate brightness stage in 1998-2001, the star went into a higher excitation Ofpe/WN9-like spectrum (Fig. 10). The spectrum is closest to those of WN 10-11 stars, which were introduced by Smith et al. (1994) and studied extensively in other papers (Crowther et al. 1995a,b,c; Crowther \& Smith 1997; Bohannan \& Crowther 1999). The most similar that we could identify in the literature are the well-known LBV AGCar during minimum (Hutsemehers \& Kohoutek 1988; Stahl 1986; Smith et al. 1994), He 3-519 (Smith et al. 1994), and S 142 (Crowther \& Smith 1997). The major criteria for a very late WN 10-11 classification (Crowther \& Smith 1997) are confirmed in the spectrum of $\mathrm{v} 532$ : i) a rich low excitation nitrogen spectrum, where N II lines are brighter than those of N III; ii) narrow He I lines, faint He II $\lambda 4686$; iii) forbidden [N II] $\lambda 5755$ and [Fe III] lines; iv) the expansion velocities derived from HeI PCyg-like profiles are low, $v_{\infty}=$ $100 \div 300 \mathrm{~km} \mathrm{~s}^{-1}$ (it is $220 \mathrm{~km} \mathrm{~s}^{-1}$ in v 532); v) the presence of a nebula with a low expansion velocity on the order of a few tens of $\mathrm{km} \mathrm{s}^{-1}$ (marginally detected in this study).

The spectrum of $v 532$ is similar to the spectra of Ofpe/WN9 stars. These stars are believed to be directly related to LBVs as in fact, "dormant" LBVs. Transitions of LBV $\leftrightarrow$ Ofpe/WN9 have been observed and are well-known, for example in AG Car, or R 127 (Stahl et al. 1983; Stahl 1986). We saw the same transition in $v 532$, when during the peak of the maximum phase (fall 1992) the star presented a low excitation LBV-like spectrum (Szeifert 1996; Fabrika 2000).

The star v 532 thus has to be classified thus as an LBV object based on its spectrum, spectral, and photometrical variability. In its current state the star may join the group of WN11 stars. The nitrogen spectrum of v 532 indicates an evolved status, where nitrogen produced in the core appears at the surface.

The B 416 spectrum and its nebula age $\left(\sim 8 \times 10^{5}\right.$ years $)$ indicate an MS or pre-LBV status (hydrogen-burning) of the star (Schaerer et al. 1993). The disk-like geometry of the nebula around B 416 does not contradict the main sequence status of 
B 416. An asymmetry in the mass loss might be due to fast rotation or close binarity.

Acknowledgements. The authors thank S. N. Dodonov and A. N. Burenkov for help during the observations. S. Fabrika and O. Sholukhova are grateful to the AIP for hospitality. This work has been supported by the RFBR grants N 03-02-16341, N 04-02-16349. O. Sholukhova acknowledges support by INTAS grant YSF 2002-281.

\section{References}

Afanasiev, V. L. 1998, html://www.sao.ru/ gafan/devices/ mpfs/mpfs_main.htm

Arribas, S., Cavaller, L., Garcia-Lorenzo, B., et al. 1998b, in Fiber Optics in Astronomy III, ed. S. Arribas, E. Mediavilla, F. Watson, ASP Conf. Ser., 152, 149

Artyukhina, N. M., Goranskii, V. P., Gorynya, N. A., et al. 1995, General Catalogue of variable stars, 5 (Moscow: Kosmosinform)

Becker, T. 2002, Thesis, University of Potsdam

Becker, T., Fabrika, S., \& Roth, M. M. 2004, Astron. Nach., 325, 155

Bohannan, B., \& Crowther, P. A. 1999, ApJ, 511, 374

Calzetti, D., Kinney, A. L., Ford, H., Dogget, J., \& Long, K. S. 1995, AJ, 110, 2739

Conti, P. S. 1976, in Be and shell stars, ed. A. Sletteback (Dordrecht: Reidel), Proc. IAU Symp., 70, 447

Corral, L. 1996, AJ, 112, 1450

Crowther, P. A., Hiller, D. J., \& Smith, L. J. 1995a, A\&A, 293, 172

Crowther, P. A., Hiller, D. J., \& Smith, L. J. 1995b, A\&A, 293, 403

Crowther, P. A., Smith, L. J., Hiller, D. J., \& Schmutz, W. 1995c, A\&A, 293, 427

Crowther, P. A., \& Smith, L. J. 1997, A\&A, 320, 500

Chu, You-Hua, Chen, C.-H. Rosie, Danforth, C., et al. 2003, AJ, 125, 2098

Fabrika, S., \& Sholukhova, O. 1995, Ap\&SS, 226, 229

Fabrika, S. 2000, in Thermal and ionization aspects of flows from hot stars: observations and theory, ed. H. J. G. L. M. Lamers, \& A. Sapar, ASP Conf. Ser., 204, 57

Fabrika, S., \& Sholukhova, O. 1999, A\&AS, 140, 309

Figer, D. F., Morris, M., Geballe, T. R., et al. 1999, ApJ, 525, 759

Garcia-Segura, G., Mac Low, M., \& Langer, N. 1996, A\&A, 305, 229

Humphreys, R. M., \& Davidson, K. 1994, PASP, 106, 1025

Humphreys, R. M., \& Sandage, A. 1980, ApJS, 44, 319

Hutsemehers, D., \& Kohoutek, L. 1988, A\&AS, 73, 217

Kudritzki, R. P. 1998, in Proc. of the 8th Canary Winter School, Stellar Astrophysics for the Local Group. A first step to the Universe, ed. A. Aparicio, A. Herrero, \& F Sanchez (New York: Cambridge Univ. Press.), 149

Kurtev, R., Sholukhova, O., Borissova, J., \& Georgiev, L. 2001, Rev. Mex. Astron. Astrofis., 37, 57

Lamers, H. J. G. L. M., Bastiaanse, M. V., Aerts, C., \& Spoon, H. W. W. 1998a, A\&A, 335, 605
Lamers, H. J. G. L. M., Zickgraf, F.-I., de Winter, D., Houziaux, L., \& Zorec, J. 1998b, A\&A, 340, 117

Lamers, H. J. G. L. M., Nota, A., Panagia, N., Smith, L. J., \& Langer, N. 2001, ApJ, 551, 764

Massey, P., Bianchi, L., Hutchings, J. B., \& Stecher, T. P. 1996, ApJ, 469,647

Massey, P., Hodge, P. W., Holmes, S., et al. 2001, AAS 199th meeting, BAAS, 33, 1496

Nota, A. 1999, in Variable and Non-spherical Stellar Winds in Luminous Hot Stars, ed. B. Wolf, O. Stahl, \& A. W. Fullerton (Springer), 62

Osterbrock, D. E. 1989, Astrophysics of Gaseous Nebulae and Active Galactic Nuclei (University Science Books, Mill Valley)

Polcaro, V. F., Gualandi, R., Norci, L., Rossi, C., \& Viotti, R. F. 2003, A\&A, 411, 193

Romano, G. 1978, A\&A, 67, 291

Roth, M. M., Bauer, S.-M., Dionies, F., et al. 2000, SPIE, 4008, 277

Roth, M. M., Becker, T., Kelz, A., \& Schmoll, J. 2003, [arXiv: astro-ph/0311407]

Sabbadin, F., \& D’Odorico, S. D. 1976, A\&A, 49, 119

Schaerer, D., Meynet, G., Maeder, A., \& Schaller, G. 1993, A\&AS, 98, 523

Shemmer, O., \& Leibowitz, E. M. 1998, IBVS, N 4595

Shemmer, O., Leibowitz, E. M., \& Szkody, P. 2000, MNRAS, 311, 698

Sholukhova, O. N., Fabrika, S. N., Vlasyuk, V. V., \& Burenkov, A. N. 1997, Astron. Lett., 23, 458

Sholukhova, O. N., Fabrika, S. N., \& Vlasyuk, V. V. 1999, Astron. Lett., 25, 14

Sholukhova, O., Zharova, A., Fabrika, S., et al. 2002, Radial and Nonradial Pulsations as Probes of Stellar Physics, ed. C. Aerts, T. R. Bedding, \& J. Christensen-Dalsgaard, ASP Conf. Proc., 259, 522

Sholukhova, O., Fabrika, S., Roth, M., \& Becker, 2004, Baltic. Astron., 13, 156

Smith, R. C., Kirshner, R. P., Blair, W. P., Long, K. S., \& Winkler, P. F. 1993, ApJ, 407, 564

Smith, L. J., Crowther, P. A., \& Prinja, R. K. 1994, A\&A, 281, 833

Stahl, O. 1986, A\&A, 164, 321

Stahl, O., Wolf, B., Klare, G., et al. 1983, A\&A, 127, 49

Szeifert, T. 1996, in 33rd Liege Institute Astroph. Coll., Wolf-Rayet stars in the framework of stellar evolution, ed. J. M. Vreux, A. Detal, D. Fraipont-Caro, E. Gosset, \& G. Rauw, Liege, 459

Walborn, N. R., \& Fitzpatrick, E. L. 2000, PASP, 112, 50

Weis, K. 2003, A\&A, 408, 205

Zharova, A. 2004, private communication

Zickgraf, F.-J., Wolf, B., Stahl, O., \& Appenzeller, I. 1986, A\&A, 163, 119

Zickgraf, F.-J., Wolf, B., Stahl, O., Leitherer, C., \& Klare, G. 1985, A\&A, 143, 421

Zickgraf, F.-J. 2003, A\&A, 408, 257 